The Teenage Brain: The Stress Response and the Adolescent
Curr Dir Psychol Sci. 2013 April ; 22(2): 140-145. doi:10.1177/0963721413475445.
Brain
Russell D. Romeo
Department of Psychology and Neuroscience and Behavior Program, Barnard College of
Columbia University, New York, NY 10027
Abstract
Adolescence is a time of many psychosocial and physiological changes. One such change is how
an individual responds to stressors. Specifically, adolescence is marked by significant shifts in
hypothalamic-pituitary-adrenal (HPA) axis reactivity, resulting in heightened stress-induced
hormonal responses. It is presently unclear what mediates these changes in stress reactivity and
what impacts they may have on an adolescent individual. However, stress-sensitive limbic and
cortical brain areas that continue to mature during adolescence may be particularly vulnerable to
these shifts in responsiveness. Consequently, perturbations of the maturing adolescent brain may
contribute to the increase in stress-related psychological dysfunctions, such as anxiety, depression,
and drug abuse, often observed during this stage of development. The purpose of this review is to
describe the changes that occur in HPA function during adolescence, as well as briefly discuss the
possible ramifications of these changes on the developing brain and psychological health.

\title{
Keywords
}

Adolescence; Hypothalamic-Pituitary-Adrenal Axis; Puberty; Stress

\begin{abstract}
Stress happens. It's a fact of life. However, the type of stressors we experience and how we respond to them change throughout our life. Adolescence represents a stage in development when both of these aspects of stress are in flux. Though most of us appreciate that the nature of stressors change during adolescence, less appreciated are the unique ways in which adolescent individuals respond to stress. This review will focus on the substantial shifts in stress reactivity exhibited during adolescence, particularly in the context of hormonal responsiveness. It will also discuss the potential impact of these hormonal changes on the developing adolescent brain. The research described in this review will largely highlight the work conducted on basic animal models, as it is from these models that most of our mechanistic understanding of age-dependent changes in stress reactivity and neurobiological function are derived.
\end{abstract}

Correspondence Author: Russell D. Romeo, Ph.D., Department of Psychology and Neuroscience and Behavior Program, Barnard College of Columbia University, New York, NY 10027, Tel: (212)854-5903, Fax: (212)854-3601, rromeo@ barnard.edu. 


\section{The Stress Response}

When an individual experiences a stressor, be it physical or psychological, two hormonal systems are activated to help the individual cope with the situation. The first is mediated by the rapid actions of the sympathetic nervous system, leading to the release of epinephrine and norepinephrine into the blood stream. It is this immediate response that mediates the transient so called "fight-or-flight" reaction to stress. The second is a slower, more protracted hormonal response mediated by the hypothalamic-pituitary-adrenal (HPA) axis. This response is initiated by a group of neurons in the paraventricular nucleus of the hypothalamus (PVN), which secrete corticotropin-releasing hormone (CRH) to signal the pituitary to release adrenocorticotropic hormone (ACTH). ACTH, in turn, stimulates the adrenal glands to synthesize and secrete the glucocorticoids (i.e., cortisol in humans and corticosterone in many rodent species). Once the stressor has ended, the glucocorticoids act though negative feedback on the pituitary gland and a variety of forebrain regions, namely the hypothalamus, hippocampus, and prefrontal cortex, ultimately terminating the response by reducing the further production and release of CRH and ACTH (Herman et al., 2003; Figure 1). Thus, in the context of the HPA reaction to stress, the brain is both the initiator and a major target of the glucocorticoid response.

The glucocorticoids are responsible for many of the adaptive physiological and behavioral responses to stressors, such as mobilizing energy stores, enhancing immune reactions, and increasing learning and memory abilities. However, chronic or more prolonged exposure to these hormones can result in numerous maladaptive outcomes, including metabolic disorders and impaired immune and cognitive functions. Thus, factors that regulate HPA reactivity and the dynamics of the hormonal stress response can have important short- and long-term consequences on an individual's physiology and behavior (McEwen, 1998).

\section{Adolescent Development of the Hormonal Stress Response}

Although adolescence is marked by many neuroendocrine changes, the shifts in HPA function are more subtle than the relatively conspicuous increases in gonadal hormones associated with puberty. That is, though basal ACTH and glucocorticoid levels remain fairly stable throughout adolescence, it is the amount and duration of these hormones released in times of stress that show notable changes. Numerous studies in both male and female rats, for instance, indicate that animals on the cusp of adolescence (i.e., $\sim 30$ days of age) show significantly protracted ACTH and corticosterone responses compared to adults (i.e., > 65 days of age) following a brief exposures to a variety of stressors (e.g., foot shock, hypoxia, restraint; Goldman, Winget, Hollingshead, \& Levine, 1973; Romeo, Lee, Chhua, McPherson, \& McEwen, 2004; Romeo, Lee, \& McEwen, 2004; Vazquez \& Akil, 1993). Specifically, these hormonal stress responses can last 45-60 min longer in animals prior to adolescent maturation (Romeo, 2010a, 2010b; Figure 2). These extended hormonal responses in per-adolescent animals are different than those of neonatal pre-weanling rats (i.e., prior to 21 days of age), which usually demonstrate hypo-responsiveness to stressors (Sapolsky \& Meaney, 1986). Interestingly, these prolonged ACTH and corticosterone responses following stress do not just gradually assume their adult-like patterns as puberty and adolescence progress, but instead show relatively more abrupt shifts, with stress-induced 
ACTH responses maturing later (i.e., between 50-60 days of age) than corticosterone responses (i.e., between 30-40 days of age; Foilb, Lui, \& Romeo, 2011; Figure 3). These data indicate that each node along the HPA axis may have its own developmental trajectory during adolescence. It is important to note that recent studies in human adolescents have also reported changes in hormonal responsiveness, though not exactly paralleling the rodent studies. More specifically, boys and girls in later stages of adolescence (15-17 years old) displaying greater stress-induced cortisol levels compared to individuals in late childhood or earlier stages of adolescence (9-13 years old; Gunnar, Wewerka, Frenn, Long, \& Griggs, 2009; Stroud et al., 2009).

\section{Contributing Factors that Mediate the Adolescent Changes in Stress Reactivity}

The mechanisms that mediate these adolescent-related changes in hormonal responsiveness remain unclear. However, it appears to involve both the activation and feedback phases of the HPA response. In the context of activation, experiments have shown that neural activity in the PVN, particularly in the CRH-containing cells, is higher in adolescent than adult animals following stress (Romeo et al., 2006; Viau, Bingham, Davis, Lee, \& Wong, 2005). These data suggest that the prolonged ACTH and corticosterone responses prior to puberty may in part be driven by greater stress-induced CRH production and release. Along with these differences in activation, studies on negative feedback have shown that pre-treatment with the synthetic glucocorticoid, dexamethasone is less effective at blunting a stressinduced corticosterone response in prepubertal compared to adult rats (Goldman et al., 1973). Thus, these results would support the notion that periadolescent animals may show less glucocorticoid-dependent negative feedback on the HPA axis than adults. Future studies will need to address what cellular mechanisms mediate these putative age-dependent changes in sensitivity to negative feedback, such as differences in glucocorticoid receptor function in the brain and pituitary. To date, however, these avenues of research have been largely unexplored.

Another factor known to significantly modulate hormonal stress reactivity in adult human and non-human animals is gonadal hormones (Viau, 2002). In males, testosterone tends to reduce hormonal stress responsiveness, while in females estradiol often enhances stress reactivity (Viau, 2002). It is possible, therefore, that the substantial difference in gonadal hormones experienced by animals before and after puberty account for the changes in stress reactivity. However, non-human animal studies that have experimentally manipulated gonadal hormone levels through surgery and/or hormone replacement have shown that prepubertal animals continue to show greater stress-induced ACTH and corticosterone responses compared to adults (Romeo, Lee, Chhua, et al., 2004; Romeo, Lee, \& McEwen, 2004). These results suggest, therefore, that simple differences in gonadal hormone levels before and after puberty are not responsible for these adolescent-related changes in HPA stress responsiveness. 


\section{The Interaction of Adolescence and Stress History on Hormonal Responsiveness}

Similar to age, previous experience with stressors can also shape one's hormonal stress responsiveness (Grissom \& Bhatnagar, 2009). For example, an adult animal repeatedly exposed to the same stressor (homotypic stress) displays a habituated hormonal response compared to an adult exposed to that stressor for the first time. On the other hand, if an animal experiences the same stressor over and over again and is then hit with a novel stressor (heterotypic stress), a sensitized hormonal response is exhibited above that evoked by the novel stressor alone. These experience-dependent changes in HPA reactivity are different before and after adolescent development. In particular, homotypic stress leads to habituation in adults, but not in pre-adolescent males (Lui et al., in press), while heterotypic stress leads to a similar peak response at both ages, but a slower recovery in animals prior to adolescence (Lui et al., in press; Figure 4). It is currently unknown at what time during adolescence development these responses to homotypic and heterotypic stressors assume their adult-like patterns.

The mechanisms responsible for this age- and experience-dependent plasticity in HPA reactivity are unclear. Though the contribution of negative feedback to these experiencedependent changes has not yet been explored, recent evidence suggests that differential activation of the PVN may be involved. That is, similar to the greater activation of PVN neurons in adolescent animals following acute stress (Romeo et al., 2006), adolescent animals also demonstrate higher PVN activation after both homotypic and heterotypic stress compared to adults, suggesting greater stress-induced hypothalamic drive to the pituitary prior to adulthood (Lui et al., in press). In addition to neurobiological substrates mediating these shifts in reactivity, peripheral factors may also play a role. For instance, it is possible that the adolescent pituitary and adrenal glands are more sensitive to CRH and ACTH, respectively, than they are in adulthood, thus in part potentially contributing to their greater responsiveness during adolescence. Regardless of the mechanisms that mediate these agespecific hormonal responses, these data suggest that following an acute or repeated stressor an adolescent individual experiences greater exposure to the glucocorticoids than an adult.

\section{Stress and the Adolescent Brain: a Perfect Storm?}

As alluded to above, the brain is a major target of the glucocorticoids and these hormones are known to be potent modulators of many neurobiological processes, including neuronal plasticity (McEwen, 2007). In adults, chronic exposure to stress results in smaller and structurally less complex hippocampal and prefrontal cortical neurons. These morphological changes are also paralleled by decreases in spatial learning and attention shifting, cognitive abilities reliant upon an intact hippocampus and prefrontal cortex, respectively. Neurons in the amygdala, conversely, show stress-induced growth in adulthood, along with increased amygdala-dependent fear learning (McEwen, 2007). Importantly, these effects of stress on the adult brain are reversible, such that if animals are allowed to recover from the stressors for at least 10 days, then these parameters revert to their pre-stress levels (McEwen, 2007). 
Though we know relatively little about how stressors experienced during adolescence may influence the immediate and long-term structure and function of the brain, many factors may converge during this stage of development that may make the adolescent brain particularly vulnerable to stressors. First, recent longitudinal structural neuroimaging studies have indicated that the areas known to be the most sensitive to stress in adulthood, namely the hippocampus, prefrontal cortex, and amygdala, all continue to mature during adolescence (Giedd \& Rapoport, 2010). Second, the adolescent brain may be more responsive to the glucocorticoids than the adult brain, as a previous animal study indicated that an equivalent dose of corticosterone increased gene expression to a greater degree in the adolescent compared to adult hippocampus (Lee, Brandy, \& Koenig, 2003). Finally, due to the increases in hormonal stress reactivity described above, it would appear that these maturing and exquisitely stress-sensitive brain regions in the adolescent would be exposed to greater and more prolonged levels of glucocorticoids. Thus, like a "perfect storm", the convergence of these factors may render the adolescent brain especially vulnerable to perturbations, and hence psychological morbidities (Romeo \& McEwen, 2006).

Unfortunately, there is a scarcity of studies that directly compare the effects of stress on the adolescent and adult brain, and fewer that track whether these effects are more enduring if the stressors occur during adolescence. Thus, support for the notion that the adolescence brain may be particularly sensitive to stress has yet to be thoroughly tested empirically. A growing body of research, however, has begun to report on the effects of acute and chronic stress exposure on the structure and function of the adolescent brain. Studies in young adult rats indicated that previous exposure to chronic stress during adolescence resulted in reduced structural plasticity in the hippocampus and prefrontal cortex, and increased plasticity in the amygdala (Eiland, Ramroop, Hill, Manely, \& McEwen, 2012; Isgor, Kabbaj, Akil, \& Watson, 2004). Additional animal studies using models of social stress during adolescence (e.g., social instability, isolation) have also indicated decreases in markers of neural plasticity, such as neurogenesis and synaptic connectivity (Leussis \& Andersen, 2008; McCormick, Nixon, Thomas, Lowie, \& Dyck, 2010). These stress-induced alterations in the adolescent brain are also associated with compromised emotional function and cognitive skills (Eiland et al., 2012; Isgor et al., 2004; Leussis \& Andersen, 2008). Although from these data it would seem that the impact of chronic stress on the adolescent and adult brain is similar, one important difference may be in the reversibility of these effects. Specifically, even a month after recovery from chronic adolescent stress, some of these structural and functional changes persist (Isgor et al., 2004; Leussis \& Andersen, 2008). It would therefore appear that the effects of stress on the adolescent brain may be longer lasting when compared to the adult. Future experiments will need to directly assess this possibility, as well as explore whether there are other unique effects of stress on the structure and function of the adolescent brain.

\section{Conclusions}

The data reviewed above clearly indicate that adolescence is time of dramatic changes in HPA function and stress responsiveness. Adolescence is also a significant period of continued neural maturation, specifically within stress-sensitive limbic and cortical regions. Thus it is possible that prolonged or repeated exposure to stress may result in a heightened 
sensitivity to these stressors, ultimately leading to maladaptive neurobehavioral development. Though the physiological and psychological implications of stress on the adolescent brain are far from clear, the increases in stress-related dysfunctions during adolescence, such as anxiety, depression, schizophrenia, and drug abuse highlight the importance of a better understanding of the interaction between changes in stress reactivity and adolescent brain development.

\section{Acknowledgments}

Funding

Preparation of this article was supported in part by grants from the National Institutes of Health (MH-090224) and the National Science Foundation (IOS-1022148).

\section{Recommended Reading}

Lupien SJ, McEwen BS, Gunnar MR, Heim C. Effects of stress throughout the lifespan on the brain, behaviour and cognition. Nature Reviews Neuroscience. 2009; 10:434-45. A highly accessible review on the impact of stress on the prenatal, neonatal, adolescent, adult, and aged brain.

McCormick CM, Mathews IZ, Thomas C, Waters P. Investigations of HPA function and the enduring consequences of stressors in adolescence in animal models. Brain and Cognition. 2010; 72:73-85. A more extensive review than the one presented here on the effects of adolescent stress, particularly on behavior. [PubMed: 19616355]

McEwen, B. S. (2007). (See References). A thorough review on HPA physiology and the influence of stress on the structure and function of the brain.

Romeo, R. D. (2010b). (See References). This review provides greater detail of HPA changes during pubertal and adolescent maturation.

Spear LP. The adolescent brain and age-related behavioral manifestations. Neuroscience and Biobehavioral Reviews. 2000; 24:417-463. An extensive and accessible review on the adolescent maturation of brain and behavior. [PubMed: 10817843]

\section{References}

Eiland L, Ramroop J, Hill MN, Manely J, McEwen BS. Chronic juvenile stress produces corticolimbic dendritic architectural remodeling and modulates emotional behavior in male and female rats. Psychoneuroendocrinology. 2012; 37:39-47. [PubMed: 21658845]

Foilb AR, Lui P, Romeo RD. The transformation of hormonal stress responses throughout puberty and adolescence. Journal of Endocrinology. 2011; 210:391-398. [PubMed: 21746793]

Giedd JN, Rapoport JL. Structural MRI of pediatric brain development: what have we learned and where are we going? Neuron. 2010; 67:728-734. [PubMed: 20826305]

Goldman L, Winget C, Hollingshead GW, Levine S. Postweaning development of negative feedback in the pituitary-adrenal system of the rat. Neuroendocrinology. 1973; 12:199-211. [PubMed: 4353346]

Grissom N, Bhatnagar S. Habituation to repeated stress: get used to it. Neurobiology of Learning and Memory. 2009; 92:215-224. [PubMed: 18667167]

Gunnar MR, Wewerka S, Frenn K, Long JD, Griggs C. Developmental changes in hypothalamuspituitary-adrenal activity over the transition to adolescence: normative changes and associations with puberty. Development and Psychopathology. 2009; 21:69-85. [PubMed: 19144223]

Herman JP, Figueiredo H, Mueller NK, Ulrich-Lai Y, Ostander MM, Choi DC, Cullinan WE. Central mechanisms of stress integration: hierarchical circuitry controlling hypothalamic-pituitaryadrenocortical responsiveness. Frontiers of Neuroendocrinology. 2003; 24:151-180. 
Isgor C, Kabbaj M, Akil H, Watson SJ. Delayed effects of chronic variable stress during peripubertaljuvenile period on hippocampal morphology and on cognitive and stress axis functions in rats. Hippocampus. 2004; 14:636-648. [PubMed: 15301440]

Lee PR, Brandy D, Koenig JI. Corticosterone alters N-methyl-D-aspartate receptor subunit mRNA expression before puberty. Molecular Brain Research. 2003; 115:55-62. [PubMed: 12824055]

Leussis MP, Andersen SL. Is adolescence a sensitive period for depression? behavioral and neuroanatomical findings form a social stress model. Synapse. 2008; 62:22-30. [PubMed: 17957735]

Lui P, Padow VA, Franco D, Hall BS, Park B, Klein ZA, Romeo RD. Divergent stress-induced neuroendocrine and behavioral responses prior to puberty. Physiology and Behavior. (in press).

McCormick CM, Nixon F, Thomas C, Lowie B, Dyck J. Hippocampal cell proliferation and spatial memory performance after social instability stress in adolescence in female rats. Behavioural Brain Research. 2010; 208:23-29. [PubMed: 19900485]

McEwen BS. Stress, adaptation, and disease: allostasis and allostatic load. Annals of the New York Academy of Sciences. 1998; 840:33-44. [PubMed: 9629234]

McEwen BS. Physiology and neurobiology of stress and adaptation: central role of the brain. Physiological Reviews. 2007; 87:873-904. [PubMed: 17615391]

Romeo RD. Adolescence: a central event in shaping stress reactivity. Developmental Psychobiology. 2010a; 52:244-253. [PubMed: 20175102]

Romeo RD. Pubertal maturation and programming of hypothalamic-pituitary-adrenal reactivity. Frontiers in Neuroendocrinology. 2010b; 31:232-240. [PubMed: 20193707]

Romeo RD, Bellani R, Karatsoreos IN, Chhua N, Vernov M, Conrad CD, McEwen BS. Stress history and pubertal development interact to shape hypothalamic pituitary adrenal axis plasticity. Endocrinology. 2006; 147:1664-1674. [PubMed: 16410296]

Romeo RD, Lee SJ, Chhua N, McPherson CR, McEwen BS. Testosterone cannot activate an adult-like stress response in prepubertal male rats. Neuroendocrinology. 2004; 79:125-132. [PubMed: 15103225]

Romeo RD, Lee SJ, McEwen BS. Differential stress reactivity in intact and ovariectomized prepubertal and adult female rats. Neuroendocrinology. 2004; 80:387-393. [PubMed: 15741744]

Romeo RD, McEwen BS. Stress and the adolescent brain. Annals of the New York Academy of Sciences. 2006; 1094:202-214. [PubMed: 17347352]

Sapolsky RM, Meaney MJ. Maturation of the adrenocortical stress response: neuroendocrine control mechanisms and the stress hyporesponsive period. Brain Research Reviews. 1986; 396:64-76.

Stroud LR, Foster E, Papandonatos GD, Handwerger K, Granger DA, Kivlighan KT, Niaura R. Stress response and the adolescent transition: performance versus peer rejection stressors. Development and Psychopathology. 2009; 21:47-68. [PubMed: 19144222]

Vazquez DM, Akil H. Pituitary-adrenal response to ether vapor in the weanling animal: characterization of the inhibitory effect of glucocorticoids on adrenocorticotropin secretion. Pediatric Research. 1993; 34:646-653. [PubMed: 8284104]

Viau V. Functional cross-talk between the hypothalamic- pituitary-gonadal and -adrenal axes. Journal of Neuroendocrinology. 2002; 14:506-513. [PubMed: 12047726]

Viau V, Bingham B, Davis J, Lee P, Wong M. Gender and puberty interact on the stress-induced activation of parvocellular neurosecretory neurons and corticotropin-releasing hormone messenger ribonucleic acid expression in the rat. Endocrinology. 2005; 146:137-146. [PubMed: 15375029] 
Activation/Drive

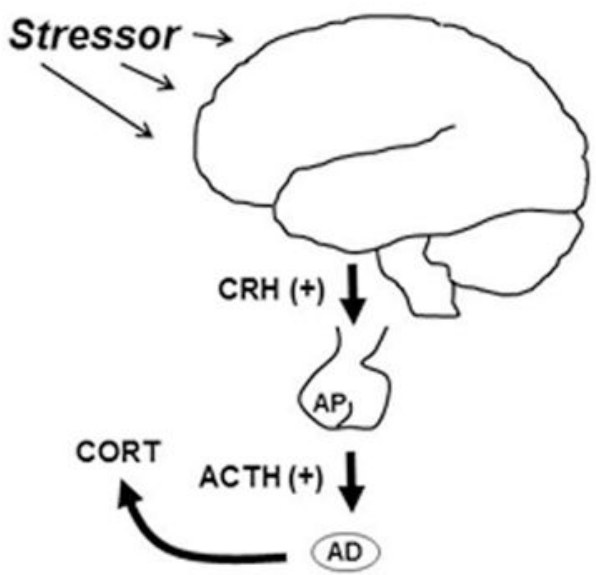

Negative Feedback/Recovery

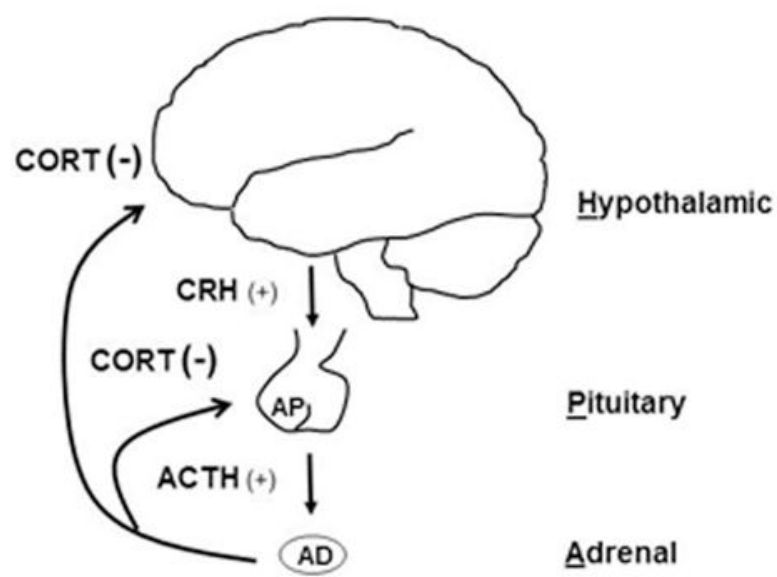

Figure 1.

A simplified schematic of stress-induced activation of the hypothalamic-pituitary-adrenal (HPA) axis as well as the negative feedback that allows the HPA axis to recover back to baseline following termination of a stressor. Abbreviation: adrenal gland (AD), adrenocorticotropin hormone (ACTH), anterior pituitary (AP), corticosterone (CORT), corticotropin-releasing hormone $(\mathrm{CRH})$, negative feedback $(-)$, positive drive $(+)$. 

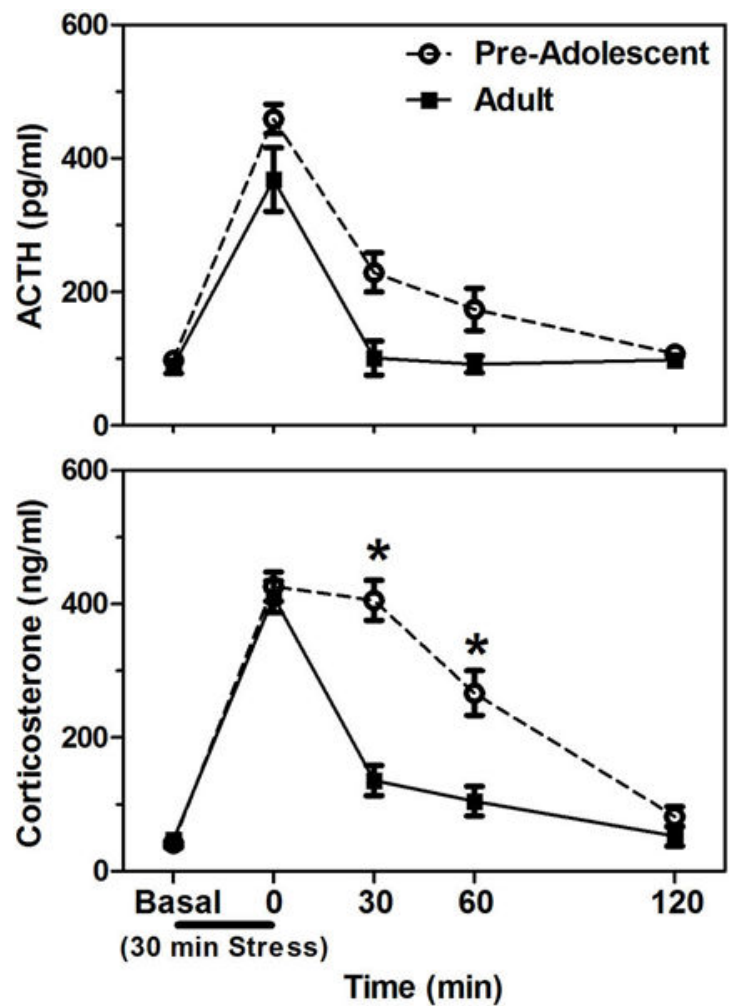

Figure 2.

Mean ( \pm SEM) plasma adrenocorticotropin hormone (ACTH) and corticosterone levels in pre-adolescent (28 days of age) and adult (77days of age) male rats before, during, and after a 30 min session of restraint stress (black bar under $\mathrm{x}$-axis). Asterisks indicate a significant difference between the ages at that time point. Adapted from (Romeo, Lee, Chhua, et al., 2004). 

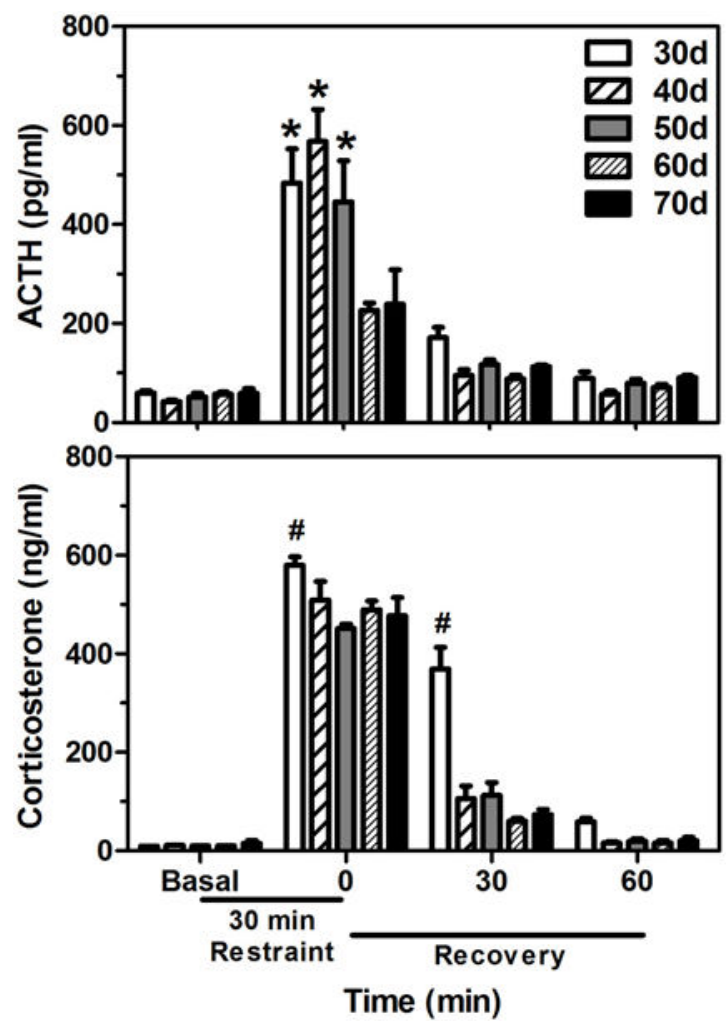

Figure 3.

Mean ( \pm SEM) plasma adrenocorticotropin hormone (ACTH) and corticosterone levels throughout pubertal and adolescent development in 30-, 40-, 50-, 60-, and 70-day old male rats before, during, and after a 30 min session of restraint stress (black bar under $\mathrm{x}$-axis). In the upper panel, asterisks indicate significant differences from the 60- and 70-day old rats at that time point. In the lower panel, “\#” indicates that 30-day old rats were significantly different from all other ages at that time point. Adapted from (Foilb et al., 2011). 
Acute Stress

Homotypic Stress

Heterotypic Stress
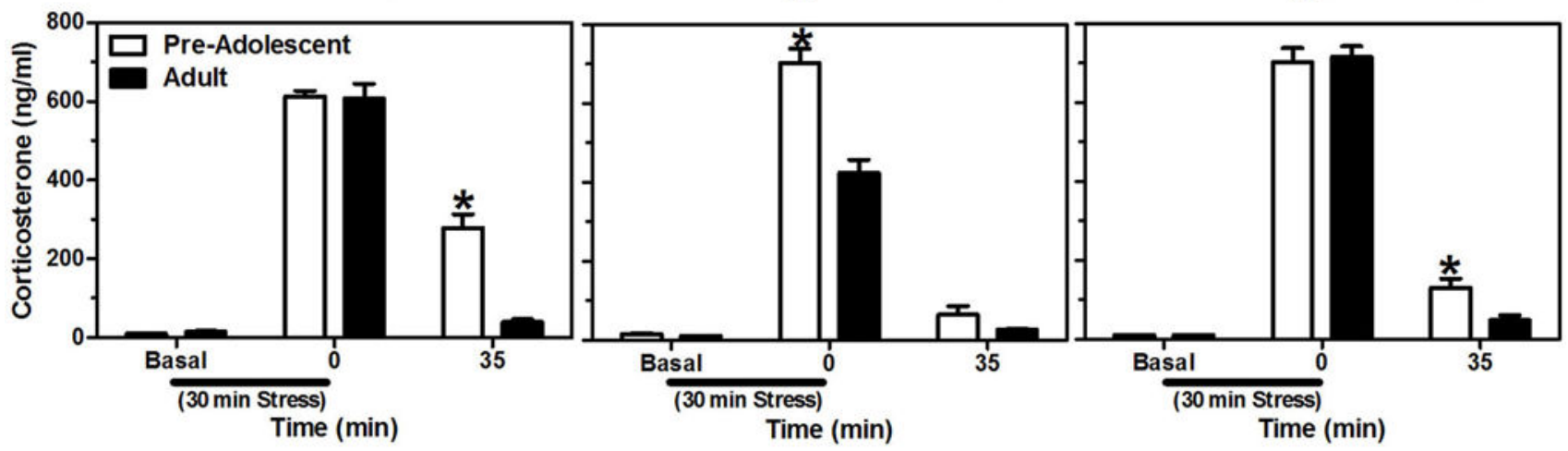

Figure 4.

Mean ( \pm SEM) plasma corticosterone levels in pre-adolescent (30 days of age) and adult (77days of age) male rats before, during, and after a 30 min session of restraint stress (black bar under x-axis) following acute, homotypic, or heterotypic stress paradigms. Acute stress consisted of a single $30 \mathrm{~min}$ session of restraint stress, homotypic stress a daily $30 \mathrm{~min}$ session of restraint stress for 8 consecutive days, and heterotypic stress a daily $30 \mathrm{~min}$ session of cold room exposure (at $4^{\circ} \mathrm{C}$ ) for 7 consecutive days followed by $30 \mathrm{~min}$ of restraint on the $8^{\text {th }}$ day. Asterisks indicate a significant difference between the ages at that time point. Adapted from (Lui et al., in press). 